\title{
A Use of Fuzzy TOPSIS to Improve the Network Selection in Wireless Multiaccess Environments
}

\author{
Mouad Mansouri $\mathbb{D}^{1}{ }^{1}$ and Cherkaoui Leghris ${ }^{2}$ \\ ${ }^{1}$ Mansouri Mouad Computer Science Department, RTM Team, FST Mohammedia, Hassan II University Casablanca, \\ Morocco BP 146, Mohammedia 220650, Morocco \\ ${ }^{2}$ Leghris Cherkaoui Computer Science Department, RTM Team, FST Mohammedia, Hassan II University Casablanca, \\ Mohammedia 220650, Morocco \\ Correspondence should be addressed to Mouad Mansouri; mansouri.mouad@yahoo.com
}

Received 26 January 2020; Revised 8 April 2020; Accepted 10 June 2020; Published 1 July 2020

Academic Editor: Juraj Machaj

Copyright (C) 2020 Mouad Mansouri and Cherkaoui Leghris. This is an open access article distributed under the Creative Commons Attribution License, which permits unrestricted use, distribution, and reproduction in any medium, provided the original work is properly cited.

Constantly faster, mobile terminals are developing, as well as wireless networks, to satisfy the growth of "Always Best Connected" demand. Users nowadays want to access the best available wireless network, either from 3GPP or IEEE group technologies, wherever they are, without losing their sessions. Consequently, mobile terminals must seamlessly transfer the communications to another access technology (vertical handover) if needed, as they often move into heterogeneous wireless environments. This work aims to optimize the network selection step in the vertical handover process. Multiattribute Decision-Making methods naturally fit this context. Nevertheless, they make wrong handover decisions sometimes, due to imprecise data collected from the metrics. This manuscript presents the use of a hybrid method, combining the fuzzy technique for order preference by similarity to the ideal situation and fuzzy analytic network process, in the network selection, to improve the quality of service and avoid, as much as possible, unnecessary handovers. The results demonstrate that this combination is the best, compared to the other methods of the same type in the network selection context.

\section{Introduction}

The demand for anywhere and anytime wireless access grows with the development of connectable devices and mobile networks, principally 3 GPP group technologies (i.e., GPRS, UMTS, etc.) and the IEEE group, including 802.11 (WLAN) and 802.16 (WiMAX). In fact, users want to connect always with reasonable quality, so they could access all the services they claim anytime without interruptions. Now that wireless technologies are developing quickly, mobile terminals (MTs) such as computers, smartphones, or tabs integrate multiple interfaces, allowing them to access the best available network anytime and anywhere. This capacity often leads to wireless access type change. This transfer's name is vertical handover $(\mathrm{VH})$, unlike the horizontal one $(\mathrm{HH})$, which means the base station or access point switch, without changing the wireless technology. Furthermore, the VH must be "seamless", which requires an efficient and fast enough $\mathrm{VH}$ strategy, not to cause session ruptures every time when a VH occurs [1].

Media Independent Handover (MIH) or IEEE 802.21 outlines the $\mathrm{VH}$ phases and provides through MIH Function (MIHF) the events, the triggers, and the service messages, to manage effectively the $\mathrm{VH}$ process. The $\mathrm{VH}$ can be terminal or network-initiated. In our work, we suppose that the VH is terminal-initiated, but network-assisted, which means that the MT decides if it should transfer the communications to a newly found network and launches the connection switch, based on the local metrics and information received from the network side via MIHF [2].

MIH divides the VH process up into three main stages, which are the $\mathrm{VH}$ initiation, preparation, and execution (Figure 1). The VH preparation is a transitional step, during which the MT sets up different parameters, to begin the communication transfer. MIH's scope excludes the 


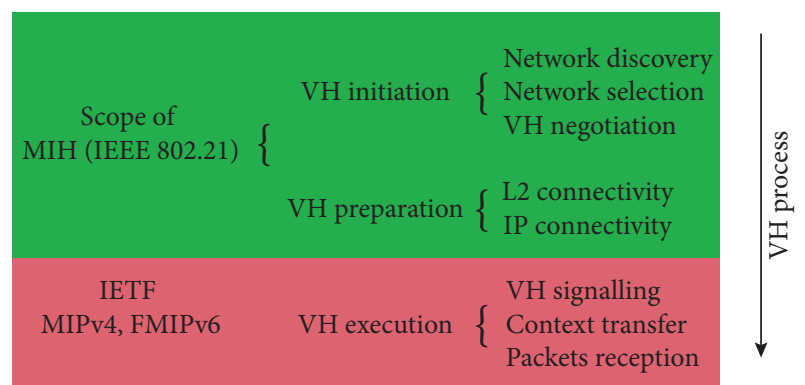

Figure 1: Steps of the general VH process.

execution, and IETF makes the standards for this stage. We assume for a seamless $\mathrm{VH}$ that the connection transfer type is "make before break" $[2,3]$, which ensures the session continuity. The initiation stage is our main concern in this work, which, in turn, runs three steps [2]:

(1) Network discovery: at this step, the MT scans the available wireless networks around its location and gathers their different metrics, which will be the attributes' values for every alternative

(2) Network selection: data gathered in the first step are organized, normalized, and computed, to score the alternatives and designate the best one, seeing all the considered attributes together

(3) $\mathrm{VH}$ negotiation: after selecting the best wireless network, the MT evaluates the necessity/utility to execute the handover before preparing and performing the communication transfer in the next steps

The network selection is a debated subject; it requires continuous optimization while maintaining a minimal execution time. We decided to focus on this step in our works regardless of the other $\mathrm{VH}$ steps. The works tackling the network selection in the literature were considering a single attribute, such as received signal strength (RSS), or the bandwidth [4]. However, this does not satisfy the user's needs seeing other important criteria, such as the cost, the security, and energy consumption of the selected access network. Some works used aggregation methods that consider more than one attribute in decision-making, such as cost and utility functions. We found in the literature more complex theories, applied in the network selection context, such as fuzzy logic [5], gaming theory [6], neural networks, and Multiattribute Decision-Making (MADM) methods [7]. Other works propose hybrid methods that combine more than one theory to make the decision in different contexts [8-11]. MADM methods are the best match techniques, for the network selection problem, because they can consider multiple attributes in the decision. We present in this work a hybrid method combining the fuzzy logic and the Technique for Ordering Preferences by Similarity to Ideal Solution (TOPSIS) and fuzzy analytic network process (FANP) for weighting the attributes to enhance the network selection. We compared, through simulation, its performance with other MADM combinations in the network selection, principally using TOPSIS, gray relational analysis (GRA), and fuzzy GRA (FGRA), which are efficient in the same context.

We organize this paper as follows. Section 2 will present the background of our contribution; Section 3 will be about MADM methods and their steps. In Section 4, we describe our simulation methodology. We will illustrate and discuss the results in Section 5 and we will finally conclude and give prospects and possible enhancement ways.

\section{Background}

The VH architectures aim to select the best available network, allowing mobile users (MUs) to access the different services they want anytime, anywhere they are. In fact, this decision should consider different parameters and needs continuous optimization to enhance the delivered QoS to the end-users and not to cause session ruptures. In [12], the authors present a classification of the existing architectures dealing with the mobility management in IPv4, as well as in IPv6 environments, following the used techniques to solve this issue, and they list the measurements and desired criteria to consider in comparing these strategies. In [13], the authors present a survey of network selection approaches, such as basic schemes, based on one metric, cost and utility functions that aggregate more than one attribute, MADM methods, neural network algorithms, and context-aware strategies. They also outline the requirements for developing enhanced architectures, which could minimize the signaling cost and packet delay for real-time applications. In [14], the authors present a context-aware, cross-layer, and interactive mobility management architecture, with resource allocation differentiation following the required QoS classes, for a seamless $\mathrm{VH}$ for users and services, in a heterogeneous wireless environment (i.e., including 3GPP and IEEE networks). They also give the requirements and needs for the future works treating the network selection issues. In [15], the authors describe the $\mathrm{VH}$ process and its steps, present some parameters used to select the best available wireless network in a heterogeneous environment, classify the $\mathrm{VH}$ strategies based on the used techniques, and list the different criteria to consider when evaluating them. They finally propose some combinations of techniques that could make better decisions and allow the best connectivity.

MADM methods, also cited in the literature as Multicriteria Decision-Making (MCDM) methods, are used to select the best one from a set of candidate alternatives, seeing multiple weighted attributes. The authors in $[3,7]$ present a review and a classification of the most significant MADM algorithms in the context of network selection in heterogeneous wireless environments, focusing on the positive and negative points of each decision-making scheme. They also discuss the attributes' choice and its impact on decisionmaking quality. They finally give an overview of the current research trend in the application of MADM algorithms in the network selection context. In [16], the authors distinguish between three MADM groups: normalization, weighting, and ranking methods. They compare several combinations of TOPSIS, GRA, Simple Additive Weighting (SAW), and Multiplicative Exponential Weighting (MEW) 
for ranking, with different weighting and normalization methods, in the network selection in a wireless multiaccess environment. They conclude that TOPSIS and FANP perform the best, followed by GRA using the Sum normalization and FANP. They also concluded that fuzzy weighting techniques are better because they consider the uncertainties and vagueness in the judgments. The authors of [9] use fuzzy GRA (FGRA) in the VH context; they conclude that FGRA enables the MT to make steadier decisions, minimizing the overall VH number, while avoiding unnecessary VHs.

In [17], the authors present a novel approach for the design of a multicriteria network selection algorithm in heterogeneous wireless networks, to achieve seamless mobility while maximizing end-user's satisfaction. They propose a two-module selection scheme: the first one estimates the necessity of handovers and the other determines the best network for future connection using fuzzy TOPSIS (FTOPSIS) as a weighting method, based on the judgments of multiple decision-makers. They compare the results for a single-user scenario seeing two traffic classes, namely, conversational and streaming while omitting the delivered QoS evaluation of the selected networks. The authors of [18] propose a network selection algorithm that combines two MADM methods, the analytic hierarchy process (AHP) and FTOPSIS, in a heterogeneous wireless environment. They used AHP to weight the criteria, and FTOPSIS to obtain the final access network scores. The authors consider WiMAX, UMTS, and two WLANs, coexisting in the simulation environment as candidate wireless networks serving three application classes in one decision point. They compare the results according to the requirements of three applications. The authors in [19] propose a decision-making scheme for the network selection problem by combining fuzzy logic and TOPSIS. They use multiple fuzzy subsystems in parallel to normalize the metrics sensed from three wireless networks, namely, WWAN, WMAN, and WLAN. They use the TOPSIS algorithm to compute the parameters and rank the candidate networks. The proposed strategy shows enhancements seeing the number of handovers and handover failure rate. Nevertheless, they do not evaluate the QoS of the different chosen networks. In [20], the authors propose a novel network selection method based on the utility function and a modified version of FTOPSIS that considers the network conditions and the user preferences, as well as the QoS, to choose the network that achieves the best balance between performance and energy consumption with minimal user involvement. This technique uses trapezoidal fuzzy numbers to express the attributes' values. The authors consider in the simulation one decision point, with three wireless networks, WiMAX, WLAN, and UMTS. The authors in [21] use the analytic network process (ANP) for weighting, and a fuzzy version of the TOPSIS method for ranking, using trapezoidal fuzzy sets for the different metrics. They propose a performance evaluation of this method in different cases, and they discuss the results in comparison with TOPSIS and Fuzzy AHP-ELECTRE (FAE), considering the number of services required by users in five communication classes. The authors propose in [22] a new network selection scheme, combining the fuzzy logic and a new
MADM method, they named this new method "fuzzy Manhattan distance to the ideal alternative" (FMDIA), the performance of this new method is compared with those of several different other MADM and fuzzy MADM methods, and the results show that FMDIA outperforms them.

However, our use of FTOPSIS in the network selection context, our simulation environments, and our evaluation of the methods are different. Our previous work in [23] described a comparison between fuzzy TOPSIS and fuzzy GRA in the same context, and we decided to extend it in this work and provide detailed computation steps with numeric examples. We present our proposal in the next section, along with our evaluation methodology of the best MADM combination for the network selection context. In the next section, we present the use of MADM methods, with the mathematical equations used in every step, especially the ranking ones, which we will compare in our work.

\section{MADM Methods}

MADM methods are decision-making aid methods that help decision-makers to select the best one from a finite set of alternatives, in complex situations, to satisfy a global objective. They calculate an overall score for each alternative to decide what is the best among those available, considering multiple weighted attributes together. Experts use these methods to solve decision issues in many fields such as economy, R\&D, human resources, and energy. We mainly distinguish between MADM methods used for weighting the attributes and those used for ranking the alternatives and present some of them consecutively in this section.

3.1. Weighting Methods. Weighting methods are MADM methods used to weight the attributes in a decision-making problem. In other words, they determine the relative impact of every attribute on the global goal achievement. There exist different weighting methods in the literature, but the most known are ANP [24], AHP (which is a special case of ANP) [25], and their fuzzy forms, FANP and FAHP, respectively. These methods were basically used for ranking; the alternative with the highest weight is the best, but the subjectivity in the judgments is its major drawback. We detail, for example, the use of AHP in the following. The works in [26-29] detail the use of ANP, FAHP, and FANP methods.

AHP uses linguistic judgments, given by experts in a field, to express the relative importance of an attribute, compared with the others, toward the global goal achievement. The main steps of this method are as follows:

(1) Structuring the problem hierarchically, with the global objective in the first level, followed by the attributes in the second one and, finally, the alternatives in the last one. We illustrate the AHP structuration of the network selection problem in our contribution in Figure 2.

(2) Establishing the pairwise comparison matrix, with the relative importance of each attribute, using linguistic variables, which correspond to numeric values. Table 1 shows these linguistic judgments with 


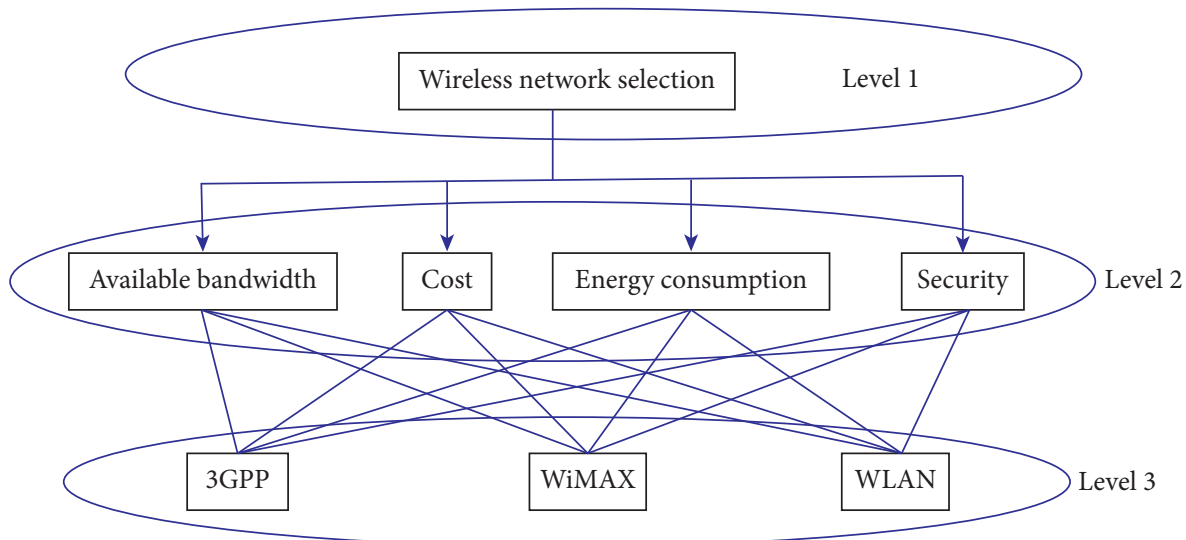

FIGURE 2: A hierarchy structuration of the network selection problem.

TABLE 1: Saaty's scale for the important judgments in the pairwise comparisons.

\begin{tabular}{lc}
\hline Saaty's scale & Relative linguistic importance of attributes \\
\hline 1 & Equally important \\
3 & Moderately important \\
5 & Strongly important \\
7 & Very strongly important \\
9 & Extremely important \\
$2,4,6,8$ & Intermediate values \\
\hline
\end{tabular}

their correspondent values, used to fill the matrix [25].

(3) Filling the pairwise comparison matrix $A_{n^{*} n}$ for $n$ attributes as in equation (1), with $a_{i j}$ being the numeric importance value of attribute $i$ toward $j, a_{j i}=1$ where $a_{i j}$ is the inverse value, and $a_{i j}(i=j)=1$ :

$$
A=\left(\begin{array}{ccc}
1 & \ldots & a_{1 n} \\
\vdots & \ddots & \vdots \\
a_{n 1} & \ldots & 1
\end{array}\right) .
$$

(4) Verifying the coherence of judgments, i.e., the Consistency Ratio (CR) of the comparison matrix must not be greater than $10 \%$ [25].

(5) Computing the matrix to obtain its Eigenvector.

(6) Normalizing and computing the Eigenvector $e v_{j}$ to have the weight vector for the attributes using

$$
W_{i}=\frac{\sum_{j=1}^{n} e v_{i j}}{n}, \quad \text { where: } \sum_{i=1}^{n} W_{i}=1 .
$$

3.2. Ranking Methods. There exist several ranking methods (TOPSIS, GRA, MEW, SAW, etc.) that follow the same main steps. They compute a decision matrix $D$, containing values of attributes for the alternatives, and assign an aggregated score for each one, considering all the weighted attributes together. These scores are then ordered, and the best alternative in the considered situation has the best score. Every
MADM method uses a different normalization and ranking technique. We present the steps of TOPSIS, modified GRA, FTOPSIS, and FGRA, respectively, as they are the main methods we will compare in our work.

3.2.1. TOPSIS. TOPSIS is one of the most used ranking methods. Like all MADM methods, TOPSIS computes the decision matrix to obtain scores for each alternative and finally rank them. We present the steps of TOPSIS in the following:

(1) Constructing the decision matrix $D_{m^{*} n}$, like in equation (3), with $d_{i j}$ being the value of an attribute $j$ for an alternative $i$. The MD collects these metrics during the discovery phase:

$$
D_{m n}=\left(\begin{array}{ccc}
d_{11} & \ldots & d_{1 n} \\
\vdots & \ddots & \vdots \\
d_{m 1} & \cdots & d_{m n}
\end{array}\right) .
$$

(2) Normalizing the decision matrix, TOPSIS uses the Euclidian normalization in equation (4), to construct the normalized decision matrix $R_{m^{*} n}$ :

$$
r_{i j}=\frac{d_{i j}}{\sum_{i=1}^{m} d_{i j}^{2}} \text {. }
$$

(3) Weighting each attribute as previously detailed in Section 3.1. Then, multiplying the weight vector $W_{j}$ by the normalized matrix $R_{m^{*} n}$ to have the weighted and normalized matrix $V_{m^{*} n}$ (equation (5)); the weight vector and the matrix must have the same lengths:

$$
v_{i j}=r_{i j} * W_{j}
$$

(4) Ranking the alternatives: TOPSIS ranking methodology is as follows:

(a) Computing the best ideal alternative $V^{+}$and the worst one $V^{-}$from the weighted and normalized matrix $V_{m^{*} n}: V^{+}=\max \left\{v_{i j}\right\}$, for benefice criteria, 
and $\min \left\{v_{i j}\right\}$, for cost criteria. $V^{-}=\min \left\{v_{i j}\right\}$, for benefice criteria, and $\max \left\{v_{i j}\right\}$, for cost criteria.

(b) Computing distances between each alternative and the best and worst ones $S^{+}$and $S^{-}$, respectively, using

$$
\begin{aligned}
& S_{i}^{+}=\sqrt{\sum_{j=1}^{n}\left(V^{+}-v_{i j}\right)^{2} .} \\
& S_{i}^{-}=\sqrt{\sum_{j=1}^{n}\left(v_{i j}-V^{-}\right)^{2} .}
\end{aligned}
$$

(c) Computing the final score $C_{i}$ for each alternative using

$$
C_{i}=\frac{S_{i}^{-}}{S_{i}^{-}+S_{i}^{+}}
$$

(d) Finally, ranking the scores $C_{i}$ for every alternative in decreasing order

3.2.2. Modified GRA. GRA is also frequently used in the network selection context. We detail the steps of this method, using a different normalization in the following.

(1) Constructing the decision matrix $D_{m^{*} n}$, like in equation (3)

(2) Normalizing the decision matrix, basically, GRA uses Min-Max but makes better decisions using the sum normalization in

$$
r_{i j}=\frac{d_{i j}}{\sum_{i=1}^{m} d_{i j}}
$$

(3) Weighting $R$ matrix using the same equation (5)

(4) Ranking: GRA ranks the alternatives as follows:

(a) Computing the best ideal alternative $V^{+}$: $V^{+}=\max \left\{v_{i j}\right\}$, for benefice criteria, and $\min$ $\left\{v_{i j}\right\}$, for cost criteria

(b) Computing the GRA coefficient (GRC) for each alternative using

$$
\mathrm{GRC}_{i}=\frac{1}{\sum_{i=1}^{n}\left|v_{i j}-V_{i}^{+}\right|+1}
$$

(c) Ranking the coefficients decreasingly

3.2.3. FTOPSIS. Starting from the conviction that fuzzy MADM methods perform better and fit more our situation, we study the use of FTOPSIS in the network selection context. The fuzzy theory consists of using fuzzy sets instead of numeric crisp values, to express the performance level of a defined attribute or a measurement. This theory introduces the notion of interval values to assess real-life judgments and nondeterministic attributes. It deals with the uncertainties in decisions and imprecision in measurements, such as in our case. We present the steps of FTOPSIS in the following:

(1) Constructing the fuzzy decision cell (FD) using equation (11), from triangular fuzzy sets instead of numeric attributes' values. We note the cell $F D_{m^{*} n}$ and its elements $f d_{i j}=\left(l_{i j}, m_{i j}, u_{i j}\right)$, which correspond to linguistic level values of each attribute $j$ for an alternative $i$. Table 2 shows the linguistic levels of performance used in our simulation and their corresponding fuzzy sets:

$$
F D_{m n}=\left(\begin{array}{ccc}
f d_{11} & \ldots & f d_{1 n} \\
\vdots & \ddots & \vdots \\
f d_{m 1} & \cdots & f d_{m n}
\end{array}\right)
$$

(2) Normalizing: the attributes' values are fuzzy sets, they are within the same range and have no units, and consequently, they need no normalization.

(3) Weighting each attribute in the same manner as for all MADM methods. The weight vector $W_{j}$ is multiplied by the elements of $F D_{m^{*} n}$ to have the weighted fuzzy cell noted $F V_{m^{*} n}$ of $f v_{i j}$ elements using

$$
\begin{aligned}
f v_{i j}\left(l_{f v}, m_{f v}, u_{f v}\right) & =f d_{i j}\left(l_{f d}, m_{f d}, u_{f d}\right) * W_{j} \\
& =f d_{i j}\left(l_{f d} * W_{j}, m_{f d} * W_{j}, u_{f d} * W_{j}\right) .
\end{aligned}
$$

(4) Ranking: the ranking methodology of FTOPSIS is as follows:

(a) Determining the best ideal alternative $F V^{+}$and the worst one $F V^{-}$from the weighted and normalized cell $F V_{m^{*} n}: F V^{+}=\max \left\{f v_{i j}\right\}$, for benefice criteria, and $\min \left\{f v_{i j}\right\}$, for cost criteria. $F V^{-} \min$ $\left\{f v_{i j}\right\}$, for benefice criteria, and $\max \left\{f v_{i j}\right\}$, for cost criteria.

(b) Distance computing: FTOPSIS computes the geometric distance between two fuzzy sets as follows: let $v_{i j}\left(l_{i j}, m_{i j}, u_{i j}\right)$ and $V_{i j}\left(L_{i j}, M_{i j}, U_{i j}\right)$ be two fuzzy sets. The distance between them is computed using

$$
d(v, V)=\sqrt{\frac{1}{3} \sum_{i=1}^{n}\left(l_{i j}-L_{i j}\right)^{2}+\left(m_{i j}-M_{i j}\right)^{2}+\left(u_{i j}-U_{i j}\right)^{2}} .
$$

So, equations (6) and (7) used to compute the distances in TOPSIS will become equations (14) and (15), and equation (13) is used to compute the distances between every element $f v_{i j}$, and $F V_{j}^{+}$and $F V_{j}^{-}$, respectively: 
TABLE 2: Fuzzy sets corresponding to linguistic levels used to assess the attributes every time to construct the $F D_{m^{*} n}$.

\begin{tabular}{lc}
\hline Linguistic variables & Fuzzy sets \\
\hline Very poor (VP) & $(1,1,2)$ \\
Poor (P) & $(1,2,3)$ \\
Medium poor (MP) & $(2,3,4)$ \\
Fair (F) & $(3,4,5)$ \\
Medium good (MG) & $(4,5,6)$ \\
Good (G) & $(5,6,7)$ \\
Very good (VG) & $(7,8,8)$ \\
\hline
\end{tabular}

$$
\begin{aligned}
& F S_{i}^{+}=\sum_{j=1}^{n} d\left(f v_{i j}, F V_{j}^{+}\right), \\
& F S_{i}^{-}=\sum_{j=1}^{n} d\left(f v_{i j}, F V_{j}^{-}\right) . \\
& F C_{i}=\frac{F S_{i}^{-}}{F S_{i}^{-}+F S_{i}^{+}} .
\end{aligned}
$$

(c) Computing the final score $F C_{i}$ for each alternative using

(d) Finally, ranking the obtained scores $F C_{i}$ for every alternative decreasingly.

3.2.4. FGRA. We describe the steps of the FGRA method in the following:

(1) Constructing the FD cell using the same equation (11).

(2) Normalizing and weighting: the FD cell needs no normalization. The same equation (12) is used for weighting the attributes.

(3) Determining the ideal alternative: $\mathrm{FV}^{+}=\max \left\{f v_{i j}\right\}$, for benefice criteria, and $\min \left\{f v_{i j}\right\}$, for cost criteria.

(4) Computing FGRA coefficient FGRC: the distance $\left|v_{i j}-V_{j}^{+}\right|$in equation (10) is calculated in FGRA, between two fuzzy sets $v_{i j}\left(l_{i j}, m_{i j}, u_{i j}\right)$ and $V_{i j}\left(L_{i j}, M_{i j}\right.$, $\left.U_{i j}\right)$, using

$$
\left|v_{i j}-V_{i j}\right|=\left|l_{i j}-L_{i j}\right|+\left|m_{i j}-M_{i j}\right|+\left|u_{i j}-U_{i j}\right| \text {. }
$$

After the fuzzy distances are calculated, they are inserted into equation (10) to compute the final FGRA coefficients $F G R C_{i}$.

(5) Finally, ranking the alternatives by their scores $F G R C i$ in decreasing order.

\section{Our Contribution}

From our perspective, MADM methods naturally fit the network selection problem, because their implementation is simple and they do not require many computational resources. Even more, they consider numerous attributes in the decision-making and their execution time is brief, which are very important and desired qualities, to perform a "seamless" VH. Nevertheless, these methods have serious drawbacks related to the frequent changes and imprecision in the metrics. The fuzzy logic deals with these uncertainties in the measurements and human judgments. We study in this work the use of FTOPSIS, with FANP for weighting the attributes, to enhance the network selection process. We propose a simulation to compare this combination with the best normal MADM methods, as well as with FGRA in the same context.

We based our simulation on the use case scenario in [2]. The scenario describes a movement of an MU, who comes home from outside, stays for a moment, and then leaves to the airport, through different "decision zones" (DZs), where heterogeneous wireless networks are available, so the MT must every time select the best one of them, using MIHF messages and considering multiple attributes together. There are four main DZs in the movement scenario, as illustrated in Figure 3, which are as follows:

(1) Before arriving home (DZ1) from $t=t_{1}$ to $t=t_{2}$

(2) The moment he stays home (DZ2) from $t=t_{2}$ to $t=t_{3}$

(3) The travel to the airport (DZ3) from $t=t_{3}$ to $t=t_{4}$

(4) Finally, the airport area (DZ4) from $t=t_{4}$ to $t=t_{5}$

We designed two datasets containing simulated discovered data, i.e., attributes' values for each available network, sensed during the discovery step, in different DZs. We used the first dataset for MADM methods simulation; it contains numeric decision matrices $D_{m^{*} n}$ from Table 3, in different DZs. We variated the input data of the available wireless technologies in a DZ, from the minimal to the maximal of the possible values, to simulate the real metrics changes. For example, when UMTS is available, the throughput in different decision points (DPs) in the same DZ varies from $144 \mathrm{kbps}$ to 2 Mbps.

For fuzzy MADM method simulation, we used the second dataset containing the fuzzy decision cells $F D_{m^{*} n}$ as described in FTOPSIS and FGRA steps. Every time a wireless access network is available, we assessed its attributes using the linguistic levels from Table 4, corresponding to triangular fuzzy numbers from Table 2 . We designed this dataset to follow the same changes as described in the use case scenario.

We considered in this simulation nine wireless networks: GPRS, EDGE, UMTS, HSXPA, LTE, Wi-Fi a/b/g, Wi-Fi $n$, Wi-Fi ac, and WiMax, and eight attributes: Throughput (T), Availability (Av), Security $(S)$, Delay $(D)$, Losses $(L)$, Cost $(C)$, Energy Consumption $(\mathrm{Ec})$, and Jitter $(J)$. We compared the results using four weighting methods, namely, AHP, ANP, FAHP, and FANP.

The next section illustrates the results of our simulation and discusses them.

\section{Results and Discussion}

We ran the simulation using different combinations and stored the decisions made during the movement (i.e., the 


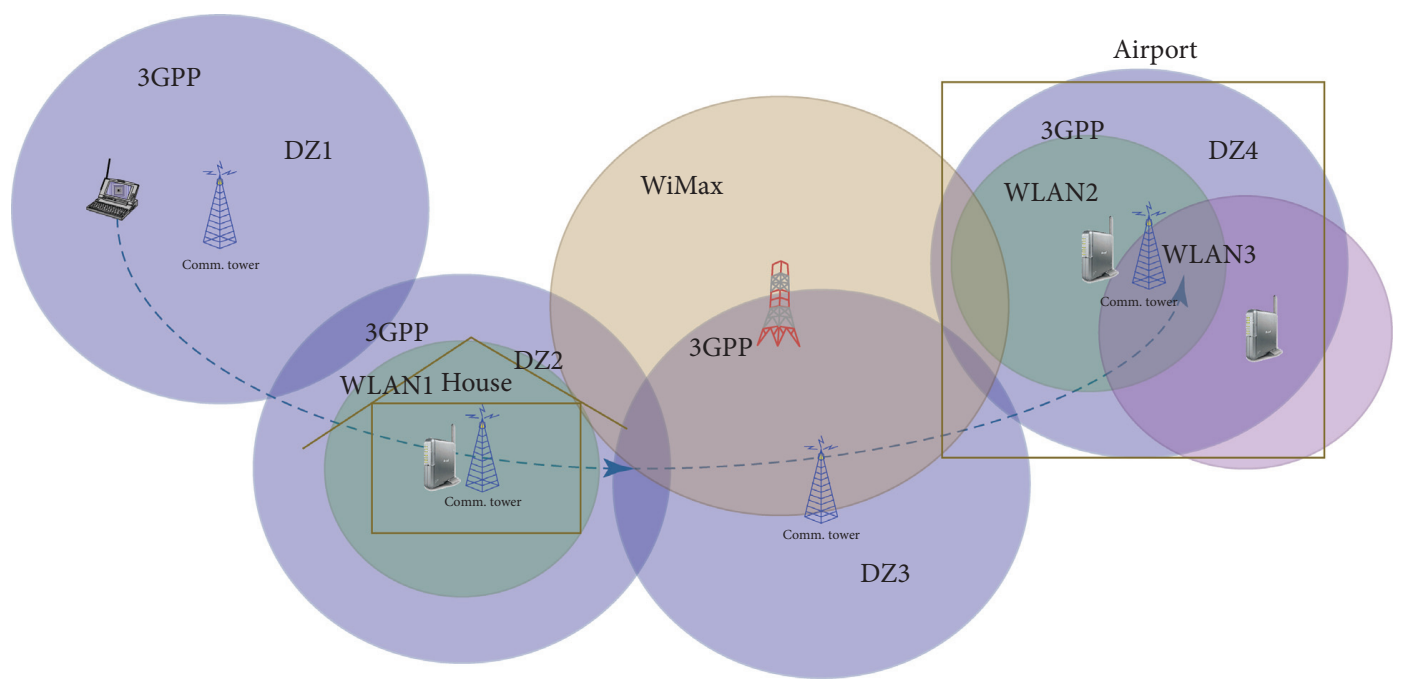

FIgURE 3: The proposed use case simulation scenario.

TABLE 3: Values of attributes used in the simulation of MADM methods.

\begin{tabular}{|c|c|c|c|c|c|c|c|c|}
\hline Network/attributes & $F$ (kbps) & Av (\%) & $S(\%)$ & $D(\mathrm{~ms})$ & $L$ & $\mathrm{EC}^{*}$ & $C^{*}$ & $J(\mathrm{~ms})$ \\
\hline GPRS & $21.4-171.2$ & $50-100$ & 50 & $50-70$ & $50-80$ & 2 & 1 & $3-20$ \\
\hline EDGE & $43.2-345.6$ & $40-100$ & 50 & $20-60$ & $25-70$ & 2 & 2 & $3-20$ \\
\hline UMTS & $144-2000$ & $40-100$ & 60 & $20-40$ & $15-65$ & 4 & 4 & $3-20$ \\
\hline HSDPA & 14 MвPS & $50-100$ & 60 & $10-50$ & $10-80$ & 5 & 5 & $3-20$ \\
\hline LTE & 10-300 MвPS & $40-100$ & 65 & $10-30$ & $10-40$ & 7 & 7 & $3-20$ \\
\hline $\mathrm{Wi}-\mathrm{Fi} a, b, g$ & 8-54 MвPs & $40-100$ & 60 & $130-200$ & $30-70$ & 3 & 1 & $3-20$ \\
\hline Wi-Fi $n$ & $72-450$ MвPS & $30-100$ & 65 & $100-140$ & $20-60$ & 4 & 1 & $3-20$ \\
\hline Wi-Fi $a c$ & $433-1300$ MBPS & $50-100$ & 70 & $90-110$ & $10-40$ & 5 & 2 & $3-20$ \\
\hline WiMax & 70 MвPS & $40-100$ & 60 & $60-100$ & $10-70$ & 7 & 5 & $3-20$ \\
\hline
\end{tabular}

* The attributes of energy consumption (Ec) and cost $(C)$ are expressed on a scale of 1 to 7 . The attributes $F, A V$, and $S$ are of benefit, and $D, L, E C, C$, and $J$ are of cost.

TABLE 4: Linguistic values of attributes used in the simulation of fuzzy MADM methods.

\begin{tabular}{lccccccc}
\hline Network/attributes & $F$ & Av & $S$ & $D$ & $L$ & EC & $C$ \\
\hline GPRS/2.5G & VP & P & F & VG & VG & P & P \\
EDGE/2.75G & P & MP & F & G & VG & P & P \\
UMTS & F & F & G & F & MG & MG & MG \\
HSDPA/HSUPA & MG & MG & G & P & F & G & F \\
LTE & G & G & VG & VP & P & VG & MP \\
Wi-Fi $a, b, g$ & F & F & MP & F & F & P & VP \\
Wi-Fi $n$ & G & MG & F & P & MP & MP & P \\
Wi-Fi ac & VG & G & MG & VP & P & F & MP \\
WiMAX & G & G & G & P & P & G & MG \\
\hline
\end{tabular}

The attributes $F, A V$, and $S$ are of benefit; $D, L, E C, C$, and $J$ are of cost.

selected networks in every DZ). We compared the results seeing the number of performed handovers, as well as the delivered QoS in each DZ. We also tested this method seeing the rank reversals number, by eliminating one alternative (the one with the last score) from the dataset, and executing the simulation again with the new parameters; if the ranking changes, we have a rank reversal. However, there is no standard performance evaluation for the proposed network selection schemes.
5.1. Results. Figure 4 illustrates the results of two MADM combinations, Euclidian normalization-TOPSIS-FANP and Sum normalization-GRA-FANP, as they are relatively the best in this context. Figure 5 shows the results using FGRA and FTOPSIS, with FANP for weighting. Figure 6 illustrates the numbers of VHs and rank reversals that occurred during the whole scenario, using different combinations.

We give numeric examples of the network selection steps, using TOPSIS and FTOPSIS with FANP in a defined 


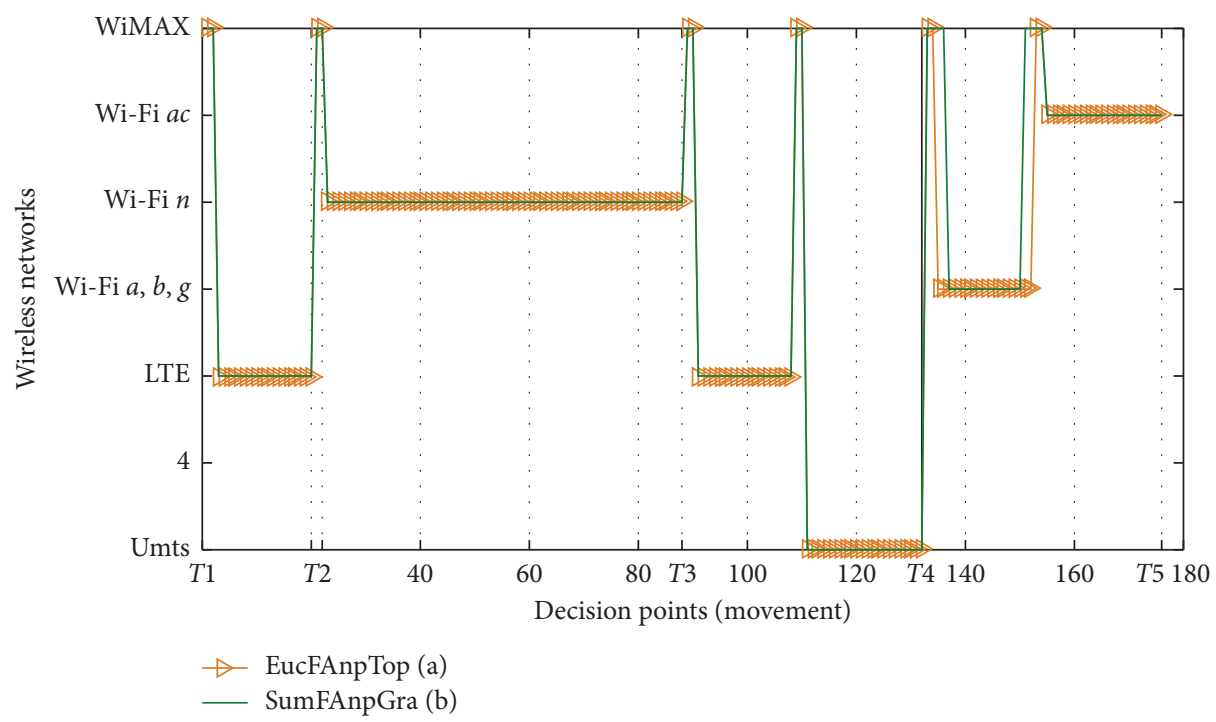

FIgURE 4: Decisions made during the movement scenario using TOPSIS-FANP (a) and Sum-GRA-FANP (b) combinations.

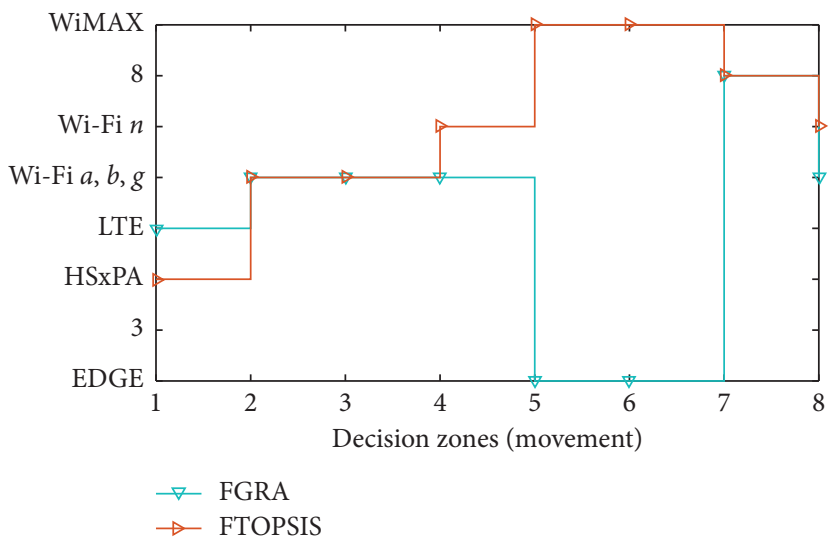

FIgURE 5: Decisions made during the movement scenario by using FTOPSIS and FGRA with FANP.

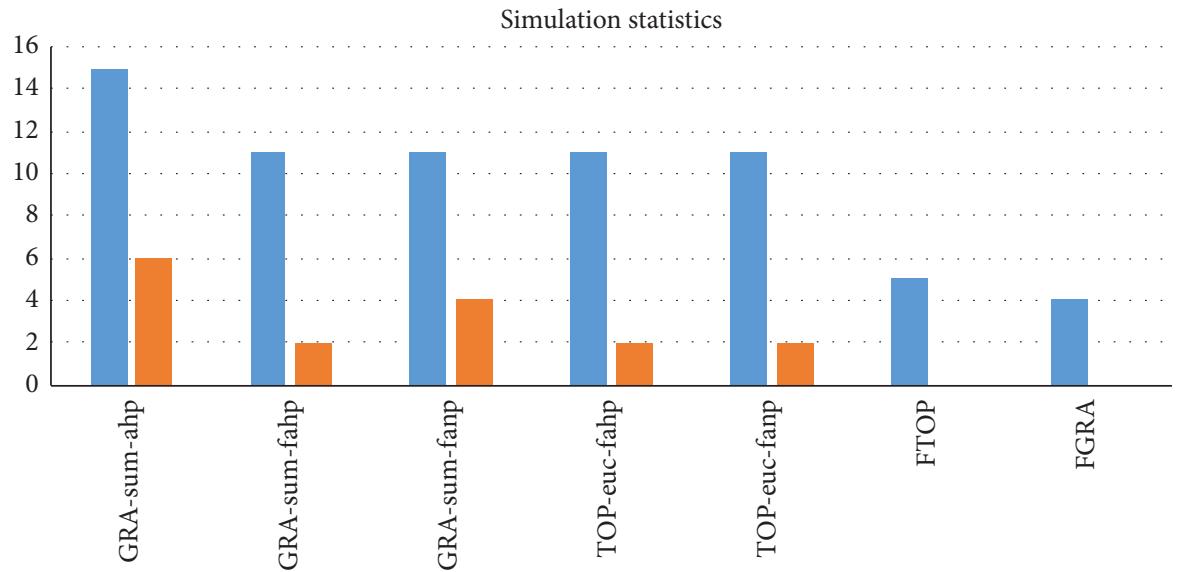

Number of $\mathrm{VH}$

- Number of rank reversals

FIgURE 6: Numbers of VHs and rank reversals performed during the simulation using each method. 
Table 5: An example of a decision matrix in a defined decision point from the dataset.

\begin{tabular}{|c|c|c|c|c|c|c|c|c|}
\hline Network/attributes & $F$ & $\mathrm{Av}$ & $S$ & $D$ & $L$ & EC & $C$ & $J$ \\
\hline GPRS/2.5G & 96.3 & 75 & 50 & 60 & 65 & 2 & 1 & 11.5 \\
\hline EDGE/2.75G & 194.175 & 70 & 50 & 40 & 47.5 & 2 & 2 & 11.5 \\
\hline UMTS & 1072 & 70 & 60 & 30 & 40 & 4 & 4 & 11.5 \\
\hline HSDPA/HSUPA & 8000 & 75 & 60 & 30 & 40 & 5 & 5 & 11.5 \\
\hline LTE & 0 & 0 & 65 & 20 & 25 & 7 & 7 & 11.5 \\
\hline $\mathrm{Wi}-\mathrm{Fi} a, b, g$ & 31000 & 70 & 60 & 165 & 50 & 3 & 1 & 11.5 \\
\hline Wi-Fi $n$ & 261000 & 65 & 65 & 120 & 40 & 4 & 1 & 11.5 \\
\hline Wi-Fi ac & 0 & 0 & 70 & 100 & 25 & 5 & 2 & 11.5 \\
\hline WiMAX & 50000 & 70 & 60 & 80 & 40 & 7 & 5 & 11.5 \\
\hline
\end{tabular}

TABLE 6: The normalized $R$ matrix.

\begin{tabular}{|c|c|c|c|c|c|c|c|c|}
\hline Network/attributes & $F$ & $\mathrm{Av}$ & $S$ & $D$ & $L$ & EC & $C$ & $J$ \\
\hline GPRS/2.5G & 0.00035 & 0.40046 & 0.27628 & 0.23457 & 0.50402 & 0.14249 & 0.08908 & 0.333 \\
\hline EDGE/2.75G & 0.00072 & 0.37376 & 0.27628 & 0.15638 & 0.36832 & 0.14249 & 0.17817 & 0.333 \\
\hline UMTS & 0.00400 & 0.37376 & 0.33154 & 0.11728 & 0.31016 & 0.28498 & 0.35634 & 0.333 \\
\hline HSDPA/HSUPA & 0.02988 & 0.40046 & 0.33154 & 0.11728 & 0.31016 & 0.35623 & 0.44543 & 0.333 \\
\hline LTE & 0 & 0 & 0.35917 & 0.07819 & 0.19385 & 0.49872 & 0.6236 & 0.333 \\
\hline Wi-Fi $a, b, g$ & 0.11581 & 0.37376 & 0.33154 & 0.64507 & 0.38771 & 0.21374 & 0.08908 & 0.333 \\
\hline Wi-Fi $n$ & 0.97508 & 0.34706 & 0.35917 & 0.46914 & 0.31016 & 0.28498 & 0.08908 & 0.333 \\
\hline Wi-Fi ac & 0 & 0 & 0.38680 & 0.39095 & 0.19385 & 0.35623 & 0.17817 & 0.333 \\
\hline WiMAX & 0.18679 & 0.37376 & 0.33154 & 0.31276 & 0.31016 & 0.49872 & 0.44543 & 0.333 \\
\hline
\end{tabular}

TABLE 7: The weighted and normalized $V$ matrix and the final scores $\$ C \_$i $\$$ using TOPSIS.

\begin{tabular}{lccccccccc}
\hline Network/attributes & $F$ & $\mathrm{Av}$ & $S$ & $D$ & $L$ & $\mathrm{EC}$ & $C$ & $J$ & Final scores $\left(C_{i}\right)$ \\
\hline GPRS/2.5G & $6.54 E-05$ & 0.10054 & 0.03863 & 0 & 0 & 0.03252 & 0.01774 & 0 & 0.48453 \\
EDGE/2.75G & 0.00013 & 0.09384 & 0.03863 & 0 & 0 & 0.03252 & 0.03548 & 0 & 0.46048 \\
UMTS & 0.00073 & 0.09384 & 0.04636 & 0 & 0 & 0.06503 & 0.07096 & 0 & 0.38759 \\
HSDPA/HSUPA & 0.00543 & 0.10054 & 0.04636 & 0 & 0 & 0.08129 & 0.0887 & 0 & 0.36776 \\
LTE & 0 & 0 & 0.05022 & 0 & 0 & 0.11381 & 0.12418 & 0 & 0.04537 \\
Wi-Fi $a, b, g$ & 0.02105 & 0.09384 & 0.04636 & 0 & 0 & 0.04878 & 0.01774 & 0 & 0.50054 \\
Wi-Fi $n$ & 0.17723 & 0.10054 & 0.05022 & 0 & 0 & 0.06503 & 0.01774 & 0 & 0.8666 \\
Wi-Fi ac & 0 & 0 & 0.05408 & 0 & 0 & 0.08129 & 0.03548 & 0 & 0.31284 \\
WiMAX & 0.03395 & 0.09384 & 0.04636 & 0 & 0 & 0.11381 & 0.0887 & 0 & 0.37151 \\
\hline
\end{tabular}

DP from DZ2; the available wireless technologies in this area are 3GPP group (as they are reachable everywhere, especially in urban areas) and the home WLAN (802.11n in our scenario). When the MD reaches this point, it collects the information to construct the $D$ matrix or FD cell and compute them to select the best network as follows:

(1) TOPSIS: we present an example of the network selection process in the defined DP, using TOPSIS:

(a) Table 5 shows $D$ matrix in the considered DP.

(b) $\mathrm{D}$ matrix is normalized to have $R$ matrix; Table 6 is the corresponding $R$ matrix.

(c) $\mathrm{R}$ matrix is multiplied by the weight vector, to have $V$ matrix, which will be computed using different MADM combinations of methods. We present here the results obtained using TOPSIS with FANP. Table 7 shows $V$ matrix and the final scores. We obtained the following vector in our simulation using FANP: $W=[0.181755546$,
$0.251073041,0.139832443,0,0,0.22820763$, $0.199131339,0]$.

(2) FTOPSIS: we present an example of the network selection process using FTOPSIS in the same DP:

(a) Table 8 shows FD cell in that DP; it contains linguistic attributes' values that will be replaced by fuzzy sets in Table 9 and computed using FTOPSIS to select the best network in the same decision point. We give in this example the worst attributes values to the unavailable networks.

(b) As we mentioned before, FTOPSIS does not need to normalize the FD; it is directly weighted. Table 10 shows the final fuzzy values with the final scores for each alternative.

5.2. Discussion. As a first observation, fuzzy MADM makes steadier decisions than normal MADM methods. We note also that fuzzy MADM methods are not sensitive to small 
TABLe 8: An example of a linguistic values cell in a defined decision point from the fuzzy dataset.

\begin{tabular}{|c|c|c|c|c|c|c|c|c|}
\hline Network/attributes & $F$ & Av & $S$ & $D$ & $L$ & EC & $C$ & $J$ \\
\hline GPRS/2.5G & $\mathrm{VP}$ & $\mathrm{P}$ & $\mathrm{F}$ & VG & VG & $\mathrm{P}$ & $\mathrm{P}$ & G \\
\hline $\mathrm{EDGE} / 2.75 \mathrm{G}$ & $\mathrm{P}$ & MP & $\mathrm{F}$ & G & VG & $\mathrm{P}$ & $\mathrm{P}$ & MG \\
\hline UMTS & $\mathrm{F}$ & $\mathrm{F}$ & $\mathrm{G}$ & $\mathrm{F}$ & MG & MG & $\mathrm{F}$ & $\mathrm{F}$ \\
\hline HSDPA/HSUPA & MG & MG & G & $\mathrm{P}$ & $\mathrm{F}$ & G & $\mathrm{F}$ & MP \\
\hline LTE & G & G & VG & VP & $\mathrm{P}$ & VG & G & $\mathrm{P}$ \\
\hline Wi-Fi $a, b, g$ & $\mathrm{~F}$ & $\mathrm{~F}$ & MP & $\mathrm{F}$ & $\mathrm{F}$ & $\mathrm{P}$ & VP & $\mathrm{F}$ \\
\hline Wi-Fi $n$ & G & MG & $\mathrm{F}$ & $\mathrm{P}$ & MP & MP & $\mathrm{P}$ & MP \\
\hline $\mathrm{Wi}-\mathrm{Fi}$ ac & $\mathrm{N} / \mathrm{A}$ & $\mathrm{N} / \mathrm{A}$ & $\mathrm{N} / \mathrm{A}$ & N/A & N/A & $\mathrm{N} / \mathrm{A}$ & N/A & N/A \\
\hline WiMAX & N/A & N/A & N/A & N/A & N/A & $\mathrm{N} / \mathrm{A}$ & N/A & N/A \\
\hline
\end{tabular}

Table 9: The fuzzy decision cell filled with the corresponding fuzzy sets.

\begin{tabular}{|c|c|c|c|c|c|c|c|c|}
\hline Network/attributes & $F$ & Av & $S$ & $D$ & $L$ & EC & $C$ & $J$ \\
\hline GPRS/2.5G & {$[1,1,2]$} & {$[1,2,3]$} & {$[3,4,5]$} & {$[7,8,8]$} & {$[7,8,8]$} & {$[1,2,3]$} & {$[1,2,3]$} & {$[5,6,7]$} \\
\hline EDGE/2.75G & {$[1,2,3]$} & {$[2,3,4]$} & {$[3,4,5]$} & {$[5,6,7]$} & {$[7,8,8]$} & {$[1,2,3]$} & {$[1,2,3]$} & {$[4,5,6]$} \\
\hline UMTS & {$[3,4,5]$} & {$[3,4,5]$} & {$[5,6,7]$} & {$[3,4,5]$} & {$[4,5,6]$} & {$[4,5,6]$} & {$[3,4,5]$} & {$[3,4,5]$} \\
\hline HSDPA/HSUPA & {$[4,5,6]$} & {$[4,5,6]$} & {$[5,6,7]$} & {$[1,2,3]$} & {$[3,4,5]$} & {$[5,6,7]$} & {$[3,4,5]$} & {$[2,3,4]$} \\
\hline LTE & {$[5,6,7]$} & {$[5,6,7]$} & {$[7,8,8]$} & {$[1,1,2]$} & {$[1,2,3]$} & {$[7,8,8]$} & {$[5,6,7]$} & {$[1,2,3]$} \\
\hline Wi-Fi $a, b, g$ & {$[3,4,5]$} & {$[3,4,5]$} & {$[2,3,4]$} & {$[3,4,5]$} & {$[3,4,5]$} & {$[5,6,7]$} & {$[7,8,8]$} & {$[3,4,5]$} \\
\hline Wi-Fi $n$ & {$[5,6,7]$} & {$[4,5,6]$} & {$[3,4,5]$} & {$[1,2,3]$} & {$[2,3,4]$} & {$[2,3,4]$} & {$[1,2,3]$} & {$[2,3,4]$} \\
\hline Wi-Fi ac & {$[1,1,2]$} & {$[1,1,2]$} & {$[1,1,2]$} & {$[7,8,8]$} & {$[7,8,8]$} & {$[7,8,8]$} & {$[7,8,8]$} & {$[7,8,8]$} \\
\hline WiMAX & {$[1,1,2]$} & {$[1,1,2]$} & {$[1,1,2]$} & {$[7,8,8]$} & {$[7,8,8]$} & {$[7,8,8]$} & {$[7,8,8]$} & {$[7,8,8]$} \\
\hline
\end{tabular}

TABLE 10: Weighted fuzzy values with final fuzzy scores FCi.

\begin{tabular}{|c|c|c|c|c|c|c|c|c|c|}
\hline $\begin{array}{l}\text { Network/ } \\
\text { attributes }\end{array}$ & $F$ & Av & $S$ & $D$ & $L$ & $\mathrm{EC}$ & C & $J$ & Final scores $\left(F C_{i}\right)$ \\
\hline GPRS/2.5G & {$[0.18,0.18,0.36]$} & {$[0.25,0.25,0.50]$} & {$[0.42,0.56,0.7]$} & 0 & 0 & {$[0.22,0.45,0.68]$} & {$[0.19,0.39,0.59]$} & 0 & 0.4664 \\
\hline EDGE/2.75G & {$[0.18,0.36,0.54]$} & {$[0.50,0.75,1.00]$} & {$[0.42,0.56,0.7]$} & 0 & 0 & {$[0.22,0.45,0.68]$} & {$[0.19,0.39,0.59]$} & 0 & 0.5243 \\
\hline UMTS & {$[0.54,0.72,0.90]$} & {$[0.75,1.00,1.25]$} & {$[0.7,0.84,0.98]$} & 0 & 0 & {$[0.91,1.14,1.36]$} & {$[0.59,0.79,0.99]$} & 0 & 0.4966 \\
\hline HSDPA/HSUPA & {$[0.72,0.90,1.09]$} & {$[1.00,1.25,1.50]$} & {$[0.7,0.84,0.98]$} & 0 & 0 & {$[1.14,1.36,1.59]$} & {$[0.59,0.79,0.99]$} & 0 & 0.5283 \\
\hline LTE & {$[0.90,1.09,1.27]$} & {$[1.25,1.50,1.75]$} & {$[0.98,1.12,1.12]$} & 0 & 0 & {$[1.59,1.82,1.82]$} & {$[0.99,1.19,1.39]$} & 0 & 0.51 \\
\hline $\mathrm{Wi}-\mathrm{Fi} a, b, g$ & {$[0.54,0.72,0.90]$} & {$[0.75,1.00,1.25]$} & {$[0.28,0.42,0.56]$} & 0 & 0 & {$[1.14,1.36,1.59]$} & {$[1.39,1.59,1.59]$} & 0 & 0.6195 \\
\hline Wi-Fi $n$ & {$[0.90,1.09,1.27]$} & {$[1.00,1.25,1.50]$} & {$[0.42,0.56,0.7]$} & 0 & 0 & {$[0.45,0.68,0.91]$} & {$[0.19,0.39,0.59]$} & 0 & 0.6782 \\
\hline Wi-Fi ac & {$[0.18,0.18,0.36]$} & {$[0.25,0.25,0.50]$} & {$[0.14,0.14,0.28]$} & 0 & 0 & {$[1.59,1.82,1.82]$} & {$[1.39,1.59,1.59]$} & 0 & 0 \\
\hline WiMAX & {$[0.18,0.18,0.36]$} & {$[0.25,0.25,0.50]$} & {$[0.14,0.14,0.28]$} & 0 & 0 & {$[1.59,1.82,1.82]$} & {$[1.39,1.59,1.59]$} & 0 & 0 \\
\hline
\end{tabular}

variations of the weight vectors; although we used different weighting techniques, the results are the same. On the other hand, the best MADM combinations are those using fuzzy weighting methods; however, they still perform considerable unnecessary VHs, which leads to connection fluctuations (ping-pong effect) in some DPs.

From the results we presented in the previous section, FTOPSIS performs better than TOPSIS, modified GRA, and FGRA. FTOPSIS performed $5 \mathrm{VHs}$ and there were no rank reversals during the movement (Figure 6) against $11 \mathrm{VHs}$ and 2 rank reversals for the best MADM combination. Moreover, the results are better than those of MADM methods and FGRA together in terms of delivered QoS, although FGRA performs less VHs. We state this seeing the general connectivity and parameters of the chosen networks in every DZ; for instance, the network selected in DZ3 using FTOPSIS is WiMAX against EDGE using FGRA in the same DZ, which has lower bandwidth/throughput and might interrupt the session. Using MADM methods in the same
DZ selects LTE, UMTS, and WiMAX, which increases the $\mathrm{VH}$ number and disrupts the communication. The selected networks using FTOPSIS offer a satisfying QoS in all the trajectory, which ensures the session continuity. However, although FTOPSIS is the best among those compared, we cannot say that it is the best overall method in the $\mathrm{VH}$ context.

\section{Conclusion and Prospects}

In this work, we present the use of FTOPSIS in the network selection context, which is the most critical of the $\mathrm{VH}$ phases. This method combines fuzzy logic and TOPSIS and uses FANP for weighting; it helps to avoid the impact of imprecision and vagueness in the metrics of the available networks. To validate its performance, we compared through simulations the results of this method, with those of TOPSIS, modified GRA, and FGRA combinations, which are known for their efficiency in this context. The results show that 
FTOPSIS makes better and steadier decisions, with the best delivered QoS compared to all the other methods.

As future work, we will improve the attributes' weights to give suitable importance to each one to satisfy the desired parameters in different situations. This will help in reaching the best network seeing all the criteria to offer a better delivered QoS every time. We will try the fuzzy form of other MADM methods in the same context.

\section{Data Availability}

Data are available from the corresponding author upon request by email (mansouri.mouad@yahoo.com).

\section{Conflicts of Interest}

The authors declare that they have no conflicts of interest.

\section{References}

[1] S. Feirer and T. Sauter, "Seamless handover in industrial WLAN using IEEE 802.11k," in Proceedings of the 2017 IEEE 26th International Symposium on Industrial Electronics (ISIE), pp. 1234-1239, Edinburgh, UK, June 2017.

[2] Gupta V., IEEE 802.21 Media Independent Handover, 2008, http://www.ieee802.org/21/Tutorials/802\%2021-IEEETutorial.ppt.

[3] E. Obayiuwana and O. E. Falowo, "Network selection in heterogeneous wireless networks using multi-criteria decision-making algorithms: a review," Wireless Networks, vol. 23, no. 8, pp. 2617-2649, 2016.

[4] V. Dhakal and S. B. Shrestha, "Comparison of fuzzy rule based vertical handover with TOPSIS and received signal strength based vertical handover algorithms," in Proceedings of the IOE Graduate Conference, pp. 105-111, Kathmandu, Nepal, 2016.

[5] A. Prithiviraj, K. Krishnamoorthy, and B. Vinothini, "Fuzzy logic based decision making algorithm to optimize the handover performance in HetNets," Circuits and Systems, vol. 7, no. 11, pp. 3756-3777, 2016.

[6] R. Trestian, O. Ormond, and G.-M. Muntean, "Game theorybased network selection: solutions and challenges," IEEE Communications Surveys \& Tutorials, vol. 14, no. 4, pp. 1212-1231, 2012.

[7] M. Kassar, B. Kervella, and G. Pujolle, "An overview of vertical handover decision strategies in heterogeneous wireless networks," Computer Communications, vol. 31, no. 10, pp. 2607-2620, 2008.

[8] A. Gumus, A. Yayla, E. Çelik, and A. Yildiz, "A combined fuzzy-AHP and fuzzy-GRA methodology for hydrogen energy storage method selection in Turkey," Energies, vol. 6, no. 6, pp. 3017-3032, 2013.

[9] M. Mansouri and C. Leghris, "Using fuzzy gray relational analysis in the vertical handover process inwireless networks," in 5th International Conference on NetworkedSystems: NETYS 2017, A. El Abbadi and B. Garbinato, Eds., Springer, Berlin, Germany, pp. 396-401, 2017.

[10] G. Büyüközkan and G. Çifçi, "A novel hybrid MCDM approach based on fuzzy DEMATEL, fuzzy ANP and fuzzy TOPSIS to evaluate green suppliers," Expert Systems with Applications, vol. 39, no. 3, pp. 3000-3011, 2012.

[11] A. Kelemenis, K. Ergazakis, and D. Askounis, "Support managers' selection using an extension of fuzzy TOPSIS,"
Expert Systems with Applications, vol. 38, no. 3, pp. 27742782, 2011.

[12] S. Ferretti, V. Ghini, and F. Panzieri, "A survey on handover management in mobility architectures," Computer Networks, vol. 94, pp. 390-413, 2016.

[13] H. A. Bhute, P. P. Karde, and V. M. Thakare, "A vertical handover decision approaches in next generationwireless networks: a survey," International Journal of Mobile Network Communications \& Telematics, vol. 4, no. 2, pp. 33-43, 2014.

[14] S. Fernandes and A. Karmouch, "Vertical mobility management architectures in wireless networks: a comprehensive survey and future directions," IEEE Communications Surveys \& Tutorials, vol. 14, no. 1, pp. 45-63, 2012.

[15] L. Johal and A. Singh, "An overview of vertical handover process and techniques," Indian Journal of Science and Technology, vol. 9, no. 14, pp. 1-7, 2016.

[16] M. Mansouri and C. Leghris, "The use of madm methods in the vertical handover decision making context," in Proceedings of the International Conference on Wireless Networks and Mobile Communications (WINCOM), pp. 1-6, Rabat, Morocco, November 2017.

[17] F. Kaleem, A. Mehbodniya, K. K. Yen, and F. Adachi, “Application of fuzzy topsis for weighting the systemattributes in overlay networks," in Proceedings of the 2012 14th Asia-Pacific Network Operations and Management Symposium (APNOMS), pp. 1-6, Seoul, South Korea, September 2012.

[18] A. Sgora, P. Chatzimisios, and D. D. Vergados, "Access network selection in a heterogeneous environmentusing the ahp and fuzzy TOPSIS methods," in Mobile Lightweight Wireless Systems, P. Chatzimisios, C. Verikoukis, I.Santamara, LaddomadaM, and O. Hoffmann, Eds., Springer, Berlin, Germany, pp. 88-98, 2010.

[19] M. M. Alkhawlani, K. A. Alsalem, and A. A. Hussein, "Multicriteria vertical handover by topsis and fuzzylogic," in Proceedings of the 2011 International Conference on Communications and Information Technology (ICCIT), pp. 96-102, Aqaba, Jordan, March 2011.

[20] I. Chamodrakas and D. Martakos, "A utility-based fuzzy TOPSIS method for energy efficient network selection in heterogeneous wireless networks," Applied Soft Computing, vol. 12, no. 7, pp. 1929-1938, 2012.

[21] E. Skondras, A. Sgora, A. Michalas, and D. D. Vergados, "An analytic network process and trapezoidal interval-valued fuzzy technique for order preference by similarity to ideal solution network access selection method," International Journal of Communication Systems, vol. 29, no. 2, pp. 307-329, 2016.

[22] M. Mansouri and C. Leghris, "New Manhattan distance-based fuzzy MADM method for the network selection," IET Communications, vol. 13, no. 13, pp. 1980-1987, 2019.

[23] M. Mansouri and C. Leghris, "A comparison between fuzzy TOPSIS and fuzzy gra for the vertical handover decision making," in Proceedings of the 2017 Intelligent Systems and Computer Vision (ISCV), pp. 1-6, Fez, Morocco, April 2017.

[24] T. L. Saaty, "Fundamentals of the analytic network process-dependence and feedback in decision-making with a single network," Journal of Systems Science and Systems Engineering, vol. 13, no. 2, pp. 129-157, 2004.

[25] T. L. Saaty, "Decision making with the analytic hierarchy process," International Journal of Services Sciences, vol. 1, no. 1, pp. 83-98, 2008.

[26] M. Gunduz and B. K. Khader, "Construction project safety performance management using analytic network process (ANP) as a multicriteria decision-making (MCDM) tool," 
Computational Intelligence and Neuroscience, vol. 2020, Article ID 2610306, 11 pages, 2020.

[27] N. S. M. R. Rahman, M. A. A. Chowdhury, A.-A. N. Siraj, R. M. Rahman, R. Karim, and K. M. A. Alam, "Selection of most suitable secondary school alternative by multi-criteria fuzzy analytic hierarchy process," in International Conference on Multimedia and Network Information System, pp. 279-289, Wroclaw, Poland, September 2018.

[28] J.-W. Tang and T.-H. Hsu, "Utilizing the hierarchy structural fuzzy analytical network process model to evaluate critical elements of marketing strategic alliance development in mobile telecommunication industry," Group Decision and Negotiation, vol. 27, no. 2, pp. 251-284, 2018.

[29] S. K. Patil and R. Kant, "A fuzzy ANP-based approach for selection of knowledge management strategies to build resilient supply chain: an empirical case study," International Journal of Integrated Supply Management, vol. 10, no. 2, pp. 173-205, 2016. 